\title{
INFLUÊNCIA DO TEMPO SOBRE A SORÇÃO DE PESTICIDAS NÃO-IÔNICOS EM UM ARGISSOLO
}

\author{
MARCOS ANTÔNIO LIMA* \\ RENÊ LUÍS DE OLIVEIRA RIGITANO**
}

\begin{abstract}
Investigou-se em laboratório a influência do tempo sobre a sorção de pesticidas não-iônicos em material do horizonte A de um solo da classe Argissolo Vermelho - Amarelo Distrófico típico. Foram estudados cinco pesticidas, abrangendo ampla faixa de polaridade, expressa na forma do coeficiente de partição dos compostos entre n-octanol e água (Kow). Foram determinados os coeficientes de distribuição $(\mathrm{Kd})$ dos pesticidas nesse solo, utilizando-se os métodos de incubação e agitação. As determinações quantitativas dos pesticidas foram feitas por meio de cromatografia a líquido de alta eficiência. Os resultados mostraram que a sorção dos compostos no solo estudado, medida pelo método de incubação constitui processo relativamente lento. Verificou-se tendência ao equilíbrio de sorção somente após três dias, com exceção do composto menos polar estudado, cujo equilíbrio de sorção foi observado a partir de um dia de incubação. Pelo método de agitação verificou-se tendência ao equilíbrio de sorção após as primeiras horas de agitação para todos os compostos, sendo que os valores de $\mathrm{Kd}$, determinados por esse método, revelaram-se menores do que aqueles obtidos pelo método da incubação.
\end{abstract}

PALAVRAS-CHAVE: ALDICARBE; FIPRONIL; IMIDACLOPRIDO; TIAMETOXAM; TRIADIMENOL; SOLO; SORÇÃO; LIXIVIAÇÃO.

* Químico, Mestre em Agroquímica, Universidade Federal de Lavras (UFLA), Lavras, MG (e-mail: marcoslima@ufla.br).

** Engenheiro Agrônomo, Ph. D. em Entomologia-Inseticidas, Professor Titular, Departamento de Entomologia, UFLA, Lavras, MG (e-mail: rigitano@ufla.br). 


\section{INTRODUÇÃO}

A lixiviação de pesticidas, ou transporte desses compostos pelo fluxo de água que percola o solo, pode resultar na contaminação do lençol freático e, conseqüentemente, de recursos hídricos (RIGITANO e GOUVÊA, 1995; DOMAGALSKI e DUBROVSKY, 1992). A sorção de pesticidas pela matéria do solo representa um dos principais fatores que influenciam a sua movimentação nos poros presentes no solo, sendo que quanto menor o grau de sorção maior é o potencial de lixiviação do composto (KOSKINEN e HARPER, 1990).

A sorção de pesticidas em solos pode ser quantificada em laboratório por meio de diferentes métodos, sendo expressa pelo coeficiente de distribuição $(\mathrm{Kd})$ que tem unidade em volume/massa e reflete a partição do composto entre as fases sólida e aquosa do solo. Assim, $\mathrm{Kd}=\mathrm{Cs} / \mathrm{Ca}$, em que Cs $\left(\mathrm{mol} \mathrm{Kg}^{-1}\right)$ corresponde à sua concentração na fase sólida do solo e $\mathrm{Ca}\left(\mathrm{mol} \mathrm{L}^{-1}\right)$ à sua concentração na solução do solo em condições de equilíbrio (SCHWARZENBACH, GSCHWEND e IMBODEN., 1993).

A maioria dos estudos sobre sorção de pesticidas em solos envolve o método de agitação de amostras do solo com solução aquosa do pesticida, seguida da determinação da concentração do composto nos colóides do solo e na fase aquosa. Diversos trabalhos mostraram que o equilíbrio de sorção é atingido em poucas horas quando tal método é utilizado (FELSOT e DAHM, 1979; CARVALHO, RIGITANO e GOUVÊA, 2002; CAPRI et al., 2001). Tem-se generalizadamente assumido que os coeficientes de distribuição assim determinados refletem a distribuição dos compostos em condições de campo. No entanto, alguns trabalhos conduzidos pelo método de incubação de solos com pesticidas mostraram que o grau de sorção dos compostos estudados aumentou com o tempo de incubação e que o equilíbrio de sorção é atingido somente após alguns dias (WALKER, 1987; COX, KOSKINEN e YEN, 1998; CAPRI et al., 2001). Uma vez que o valor de Kd do pesticida representa importante parâmetro para a simulação da sua lixiviação em solos, o presente trabalho foi conduzido com os seguintes objetivos: a) investigar a influência do tempo de incubação sobre a sorção de alguns pesticidas não-ionicos, abrangendo ampla faixa de polaridade, em material do horizonte $A$ de um solo da classe Argissolo Vermelho-Amarelo Distrófico típico, e b) comparar os coeficientes de sorção dos compostos nesse solo, determinados pelos métodos de incubação e agitação.

\section{MATERIAL E MÉTODOS}

\subsection{SOLO UTILIZADO}

Utilizou-se no presente trabalho solo da classe Argissolo Vermelho-Amarelo Distrófico típico (PVA), localizado no Campus da Universidade Federal de Lavras (UFLA), Lavras-MG, em área de vegetação natural, nas seguintes coordenadas geográficas: $S=21^{\circ} 13^{\prime} 48^{\prime \prime}$ e W $=44^{\circ} 58^{\prime} 45^{\prime \prime}$. PIFFER e RIGITANO (1991) estudaram a lixiviação do aldicarbe em parcela experimental localizada a cerca de $10 \mathrm{~m}$ do ponto de coleta da amostra de solo utilizada no presente trabalho.

Após a retirada da serrapilheira, coletaram-se cerca de $15 \mathrm{~kg}$ de material do horizonte A desse solo na camada de 0 a $15 \mathrm{~cm}$ de profundidade. $O$ solo foi triturado, deixado secar ao ar por quatro dias, passado em peneira de malha $2 \mathrm{~mm}$ e acondicionado em saco plástico aberto para manter a aeração.

Uma amostra desse solo foi separada para a determinação do grau de umidade (estufa a $105^{\circ} \mathrm{C}$ ) e outra encaminhada ao departamento de Ciências do Solo da UFLA para determinação do teor de matéria orgânica. A determinação, feita pelo método Colorimétrico (VAN RAIJ et al., 1987), revelou teor de $3,7 \%$.

\subsection{PESTICIDAS UTILIZADOS}

Os pesticidas utilizados no presente trabalho (Quadro 1) foram escolhidos de forma a abranger 
ampla faixa de polaridade, expressa na forma do logaritmo do coeficiente de partição do composto entre $\mathrm{n}$-octanol e água $\left(\log \mathrm{K}_{\text {ow }}\right.$ ). A escolha desses compostos também ocorreu em função da viabilidade de sua determinação por meio de cromatografia a líquido de alta eficiência (CLAE), usando-se detetor de Ultra-Violeta (UV) para a quantificação dos compostos.

\section{QUADRO 1 - NOMES COMUNS, CLASSES, VALORES DE LOG Kow E FÓRMULAS ESTRUTURAIS DOS PESTICIDAS UTILIZADOS}

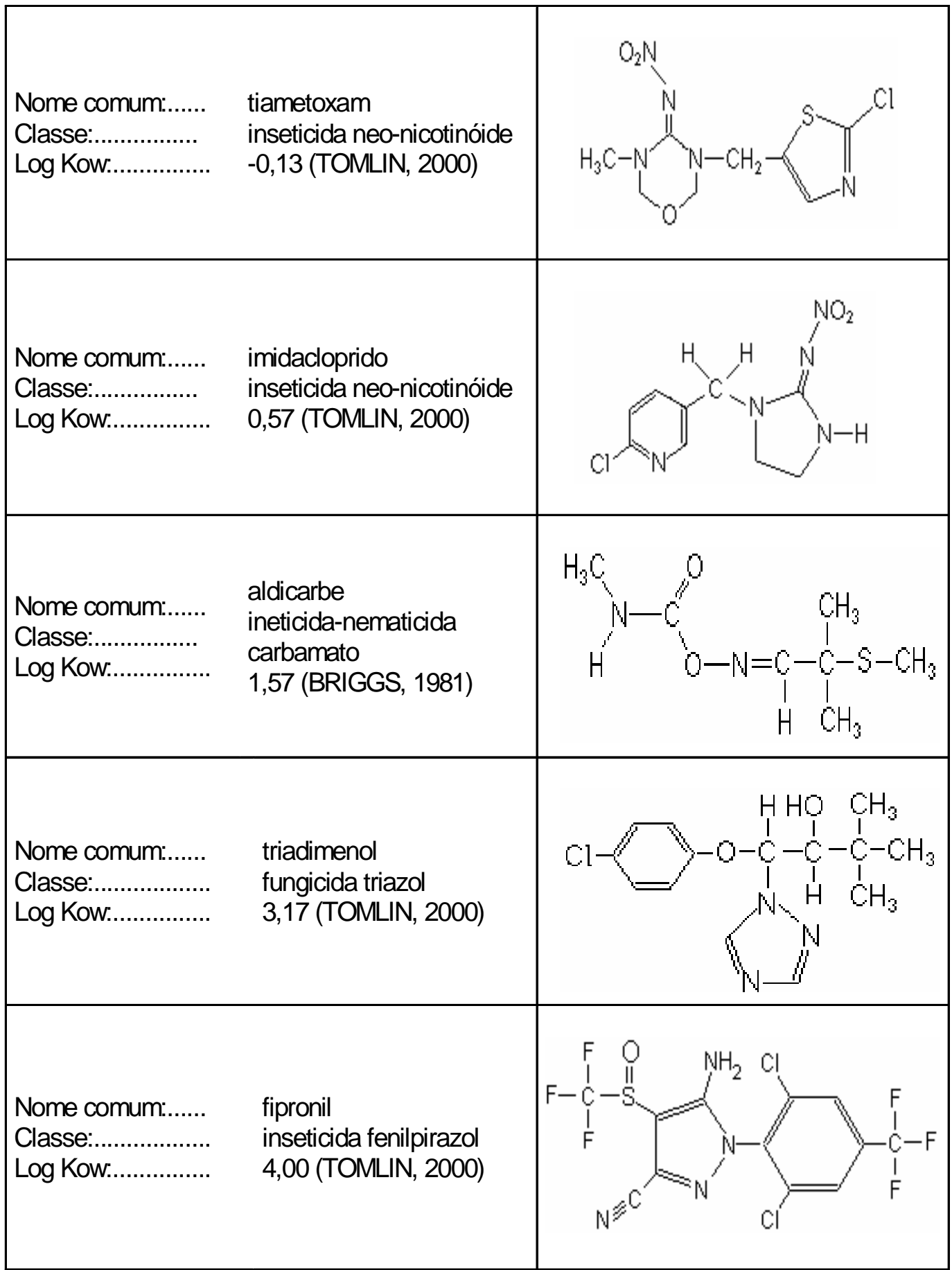




\subsection{CINÉTICA DE SORÇÃO PELO MÉTODO DE INCUBAÇÃO}

Investigou-se a cinética de sorção dos compostos pelo método de incubação, conforme metodologia descrita por CAPRI et al. (2001) com algumas modificações. Doze frascos de vidro cilíndricos de $2 \mathrm{~cm}$ de diâmetro e $7 \mathrm{~cm}$ de altura receberam 10 gramas de material de solo, aos quais aplicaram-se $5,5 \mathrm{~mL}$ de solução aquosa dos compostos com o auxílio de seringa, distribuindo-se a solução de maneira uniforme na massa de solo.

As concentrações dos compostos nas soluções aquosas foram 1, 2, 4, 8 e $40 \mu \mathrm{g} \mathrm{L}^{-1}$, respectivamente para o fipronil, imidacloprido, tiametoxam, triadimenol e aldicarbe. Essas concentrações foram definidas com base nas recomendações dos seus fabricantes para aplicação via solo na cultura do cafeeiro e, no caso do fipronil, na cultura da cana-de-açúcar. Foram consideradas as doses de ingrediente ativo recomendadas por hectare, sua aplicação em $5 \%$ da área (simulando-se a aplicação em sulcos) e a distribuição dos compostos na camada de 0 a $10 \mathrm{~cm}$ de profundidade. A quantidade de ingrediente ativo por grama de solo foi, então, calculada e utilizada para preparação das soluções aquosas dos compostos. No caso do aldicarbe utilizou-se concentração duas vezes maior do que a calculada em razão da esperada alta taxa de degradação do composto no solo, com base na literatura. Para efeito de padronização da força iônica da fase aquosa, conforme preconizado para estudos de sorção de pesticidas em solos, os compostos foram dissolvidos em água destilada contendo $0,01 \mathrm{~mol} \mathrm{~L}^{-1} \mathrm{de} \mathrm{CaCl}_{2}$ (FELSOT e DAHM, 1979).

Os frascos foram tampados com papel alumínio contendo pequenas perfurações para permitir a aeração, sendo mantidos em repouso em câmara climatizada a $25 \pm 2^{\circ} \mathrm{C}$ sem incidência de luz. A umidade do solo foi corrigida diariamente com água destilada por meio da reposição da diferença de massa com o auxílio de seringa.

Após cumprir o tempo de incubação determinado (4 e 16 horas, 1, 3, 6, e 9 dias), as amostras foram retiradas em duplicata e centrifugadas por 10 minutos a $2500 \mathrm{~g}$ no próprio frasco em que estavam acondicionadas. O sobrenadante foi retirado com pipeta Pasteur, filtrado em filtros Millipore com membrana PTFE $(0,45 \mu \mathrm{m})$ e congelado para posterior análise por cromatografia a líquido de alta eficiência (CLAE). A fase aquosa sobrenadante do composto fipronil foi misturada com acetonitrila na proporção 3:7 (água:acetonitrila), anteriormente à filtragem, pois testes preliminares revelaram que esse composto é parcialmente retido no filtro utilizado quando dissolvido somente em água.

Para a determinação da quantidade de pesticida sorvida pela amostra de solo, essa foi transferida para frasco erlenmeyer de $250 \mathrm{~mL}$. Após adição de $50 \mathrm{~mL}$ de acetona, o frasco foi mantido em mesa agitadora por uma hora e deixado em repouso por 15 minutos. Pipetou-se uma alíquota de $2 \mathrm{~mL}$ do sobrenadante, a qual foi transferida para balão de fundo redondo e esse acoplado a evaporador rotativo para eliminação da acetona. Os resíduos foram dissolvidos em $2 \mathrm{~mL}$ da mistura de acetonitrila e água, na mesmo proporção utilizada para a corrida cromatográfica para cada pesticida estudado, filtrados e congelados para posterior análise. Para melhor aproximação da quantidade de pesticida sorvida pela massa de solo deduziu-se da massa total de pesticida extraída com acetona, a massa de pesticida encontrada na solução aquosa presente no solo após a centrifugação.

Uma vez que as quantidades recuperadas dos compostos no primeiro tempo de incubação (4h) foram muito próximas àquelas aplicadas, assumiu-se eficiência de $100 \%$ para o método utilizado nas determinações das concentrações dos compostos.

Determinou-se a quantidade degradada dos compostos pela diferença entre a massa do composto aplicada inicialmente e a massa total recuperada nas fases solida e aquosa no final de cada período de incubação.

Para todos os pesticidas foram conduzidas 2 amostras adicionais com 9 dias de incubação. Determinou-se a concentração do pesticida no solo nas suas partes inferior e superior, conforme sua distribuição no frasco, para confirmação de que o pesticida estava realmente distribuído de maneira uniforme no solo e que sua sorção e degradação também ocorreram de maneira uniforme. 


\subsection{CINÉTICA DE SORÇÃO PELO MÉTODO DE AGITAÇÃO EM FRASCO}

Investigou-se a cinética de sorção dos compostos pelo método de agitação em frasco, conforme FELSOT e DAHM (1979) para efeitos comparativos com o método de incubação. Quatorze frascos erlenmeyers de $50 \mathrm{~mL}$ receberam 2 gramas de material de solo e $10 \mathrm{~mL}$ de solução aquosa dos compostos (nas mesmas concentrações utilizadas no experimento de incubação).

Os frascos foram hermeticamente fechados e levados à mesa agitadora a 140 ciclos por minuto. Após o tempo de agitação determinado (1/2, 1, 2, 4, 8, 16 e 24 horas), os frascos foram retirados em duplicata e mantidos em repouso por meia hora. Uma alíquota do sobrenadante foi coletada e centrifugada a $2500 \mathrm{~g}$ por 10 minutos. A fase aquosa foi filtrada em filtro Millipore com membrana PTFE $(0,45 \mu \mathrm{m})$ com auxílio de seringa e congelada para posterior análise da concentração do composto na solução aquosa do solo.

Calculou-se a concentração do composto na fase sólida pela diferença de massa entre a quantidade inicialmente aplicada e a quantidade encontrada na solução do solo após o período de agitação.

Uma vez que o método de agitação em frasco e o de incubação envolveram diferentes proporções de solo e solução aquosa, conduziu-se experimento adicional com a finalidade de verificar quais os efeitos dessa variação sobre os valores $\mathrm{Kd}$ dos compostos. Nesse experimento, efetuado pelo método de agitação, fixou-se o período de agitação em 24 horas e o volume utilizado para cada solução de pesticida em $10 \mathrm{~mL}$, porém as massas de solo utilizadas variaram de 0,$5 ; 1,0 ; 2,0 ;$ e $5,0 \mathrm{~g}$.

\subsection{QUANTIFICAÇÃO DOS COMPOSTOS}

As determinações quantitativas dos compostos ocorreram em sistema de Cromatografia a Líquido de Alta Eficiência, modelo Agilent série 1100, com bomba isocrática e detector ultravioleta, tendo como base os procedimentos analíticos utilizados por PIASAROLO, RIGITANO e GUERREIRO (2008). Usou-se volume de injeção de $20 \mu \mathrm{L}$ e coluna LiChroCART, preenchida com $5 \mu \mathrm{m}$ de LiChrospher RP-18, de $25 \mathrm{~cm}$ de comprimento e $3 \mathrm{~mm}$ de diâmetro interno. Utilizou-se fluxo da fase móvel de $0,3 \mathrm{~mL} \mathrm{~min}^{-1}$ para todos os compostos, porém o comprimento de onda para a detecção e a proporção de acetonitrila e água (fase móvel) variaram (Tabela 1).

TABELA 1 - CONDIÇÕES CROMATOGRÁFICAS E TEMPOS DE RETENÇÃO DOS PESTICIDAS

\begin{tabular}{lccc}
\hline Pesticidas & $\begin{array}{c}\text { Fase móvel } \\
\text { acetonitrila:água }\end{array}$ & $\begin{array}{c}\text { Comprimento } \\
\text { de onda (nm) }\end{array}$ & $\begin{array}{c}\text { Tempo de retenção } \\
\text { (min) }\end{array}$ \\
\hline Imidacloprido & $40: 60$ & 270 & 7,75 \\
Tiametoxam & $40: 60$ & 255 & 6,20 \\
Triadimenol & $70: 30$ & 235 & 8,60 \\
Fipronil & $70: 30$ & 225 & 10,80 \\
Aldicarbe & $50: 50$ & 250 & 8,62 \\
& & & \\
\hline
\end{tabular}

\section{RESULTADOS E DISCUSSÃO}

\subsection{CINÉTICA DE SORÇÃO DOS COMPOSTOS PELO MÉTODO DE INCUBAÇÃO}

A evolução dos coeficientes de sorção dos pesticidas (Kd), obtidos pelo método de incubação, é mostrada na Figura 1. Os valores de Kd do aldicarbe aos 6 e 9 dias de incubação não foram plotados 
em função da baixa quantidade remanescente do composto e elevada variação entre os valores de concentração quantificados nas repetições. Os resultados mostraram tendência ao equilíbrio somente após 3 dias de incubação para todos os compostos com exceção do fipronil, composto menos polar entre os pesticidas estudados, cuja tendência ao equilíbrio de sorção foi observada logo após 1 dia de incubação. Utilizando metodologia semelhante à empregada no presente trabalho, CAPRI et al. (2001) constataram que o equilíbrios de sorção dos compostos imidacloprido e pirimetanil foram atingidos somente após cerca de 5 dias de incubação.

\section{FIGURA 1 - EVOLUÇÃO DOS VALORES DE Kd DOS PESTICIDAS OBTIDOS PELO MÉTODO DE INCUBAÇÃO EM MATERIAL DO HORIZONTE A DE ARGISSOLO VERMELHO-AMARELO DISTRÓFICO TÍPICO}
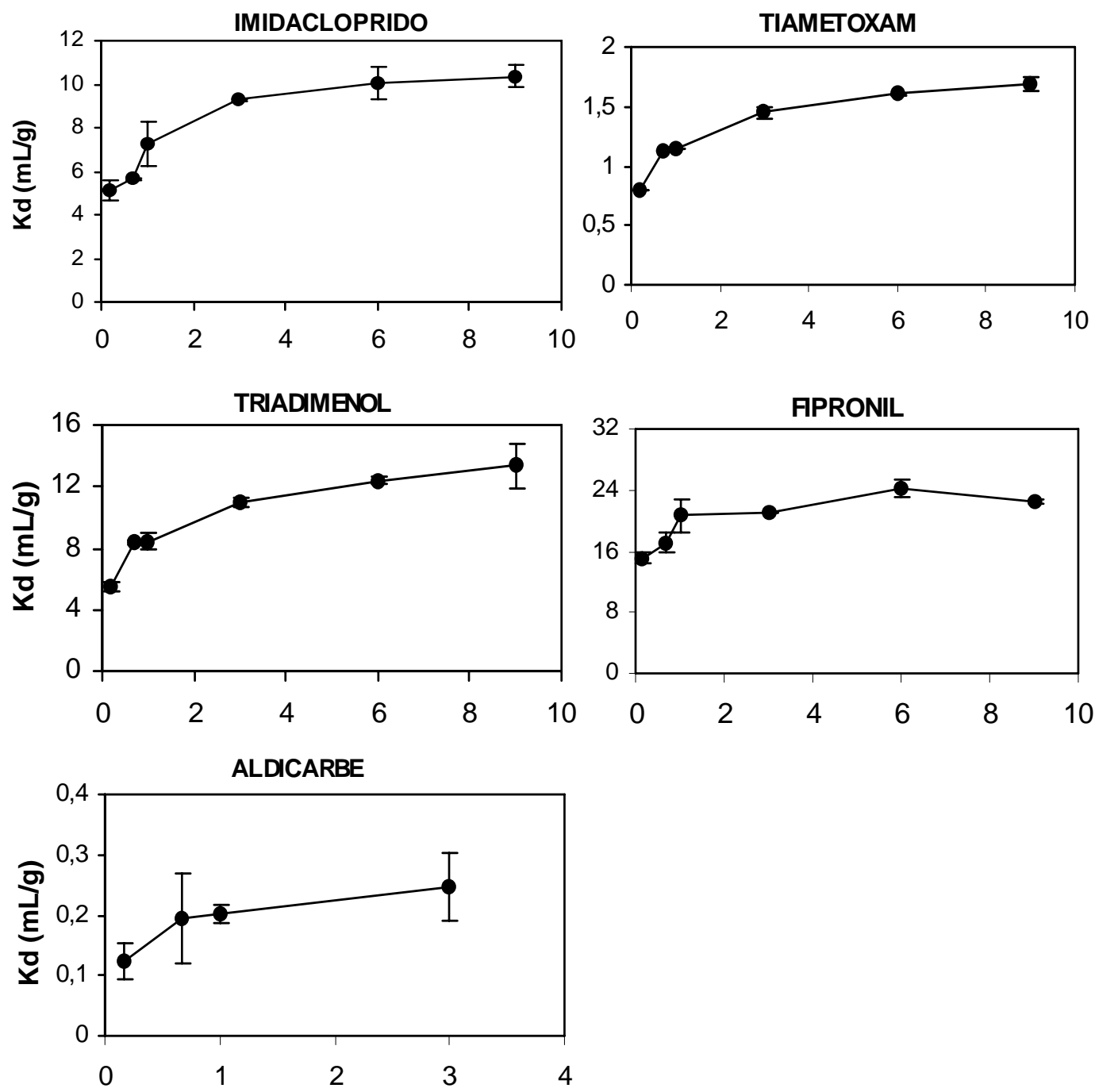

Tempo (dias)

As quantidades totais dos compostos encontradas nos frascos nos diferentes períodos de incubação, expressas em percentagens da quantidade aplicada, são apresentadas na Figura 2. Como pode ser observado, à exceção do aldicarbe, os compostos revelaram-se bastante estáveis. Examinando a correlação linear entre o logaritmo da quantidade remanescente dos compostos e o tempo de incubação encontraram-se, com exceção do triadimenol, valores de $\mathrm{R}^{2}$ maiores do que 0,7 , indicando que a degradação desses compostos seguiu cinética de primeira ordem. Os valores de meia-vida 
obtidos foram 141, 165, 433 e 5 dias, respectivamente para os compostos imidacloprido, tiametoxam, fipronil e aldicarbe. No caso do triadimenol, o valor de $R^{2}$ foi muito baixo em razão da variação ou erro experimental ter sido maior do que a variação na percentagem do composto efetivamente degradada ao longo do período de incubação.

FIGURA 2 - DEGRADAÇÃO DOS PESTICIDAS EM AMOSTRAS DE ARGISSOLO VERMELHOAMARELO DISTRÓFICO TÍPICO HORIZONTE A
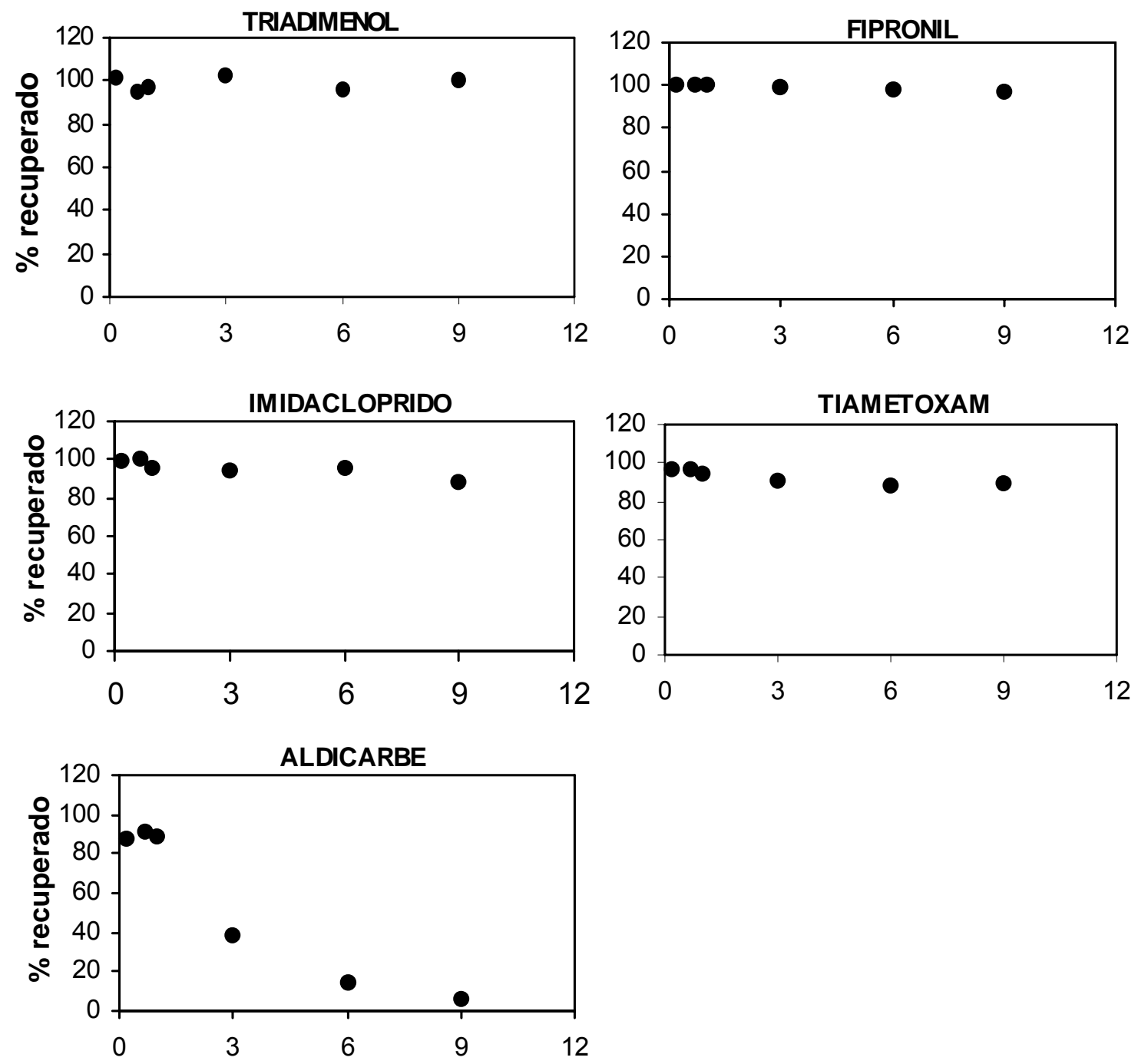

Tempo (dias)

Os resultados das análises das metades superior e inferior das amostras incubadas por 9 dias, expressos em concentração do pesticida/massa de solo ( $\mu \mathrm{g} / \mathrm{g}$ ) (Figura 3), em geral mostraram distribuição uniforme dos pesticidas no perfil do solo. Diferença maior foi observada para o aldicarbe, embora a metodologia de aplicação do pesticida no solo tenha sido a mesma para todos os compostos. Os resultados obtidos para o aldicarbe evidenciaram que a taxa de degradação desse composto na metade inferior foi maior do que na metade superior, provavelmente devido à presença de maior quantidade de água no fundo do frasco. BROMILOW et al. (1986) observaram degradação muito mais rápida do aldicarbe em condições anaeróbias e postularam que nessas condições o aldicarbe é reduzido, mediante reação catalizada por íons $\mathrm{Fe}^{2+}$. 


\section{FIGURA 3 - CONCENTRAÇÃO DOS PESTICIDAS NAS METADES SUPERIOR E INFERIOR DAS}

AMOSTRAS DE SOLO INCUBADAS COM OS COMPOSTOS POR 9 DIAS

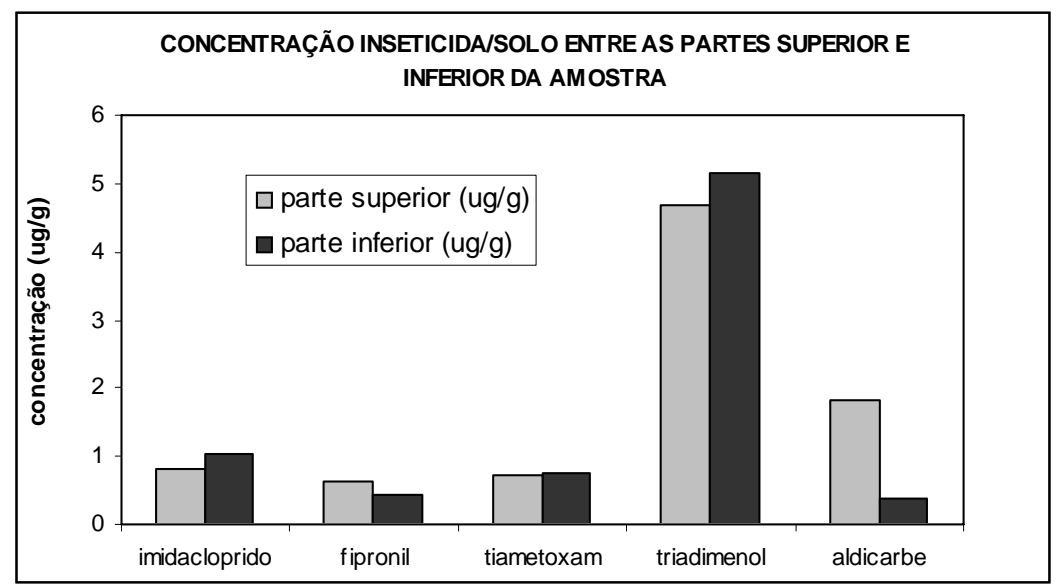

\subsection{CINÉTICA DE SORÇÃO DOS COMPOSTOS PELO MÉTODO DE AGITAÇÃO}

A evolução dos valores de Kd dos compostos em função do tempo de agitação é mostrada na Figura 4.

\section{FIGURA 4 - EVOLUÇÃO DOS VALORES DE Kd DOS PESTICIDAS OBTIDOS PELO MÉTODO DE AGITAÇÃO EM MATERIAL DO HORIZONTE A DE ARGISSOLO VERMELHO-AMARELO DISTRÓFICO TÍPICO}
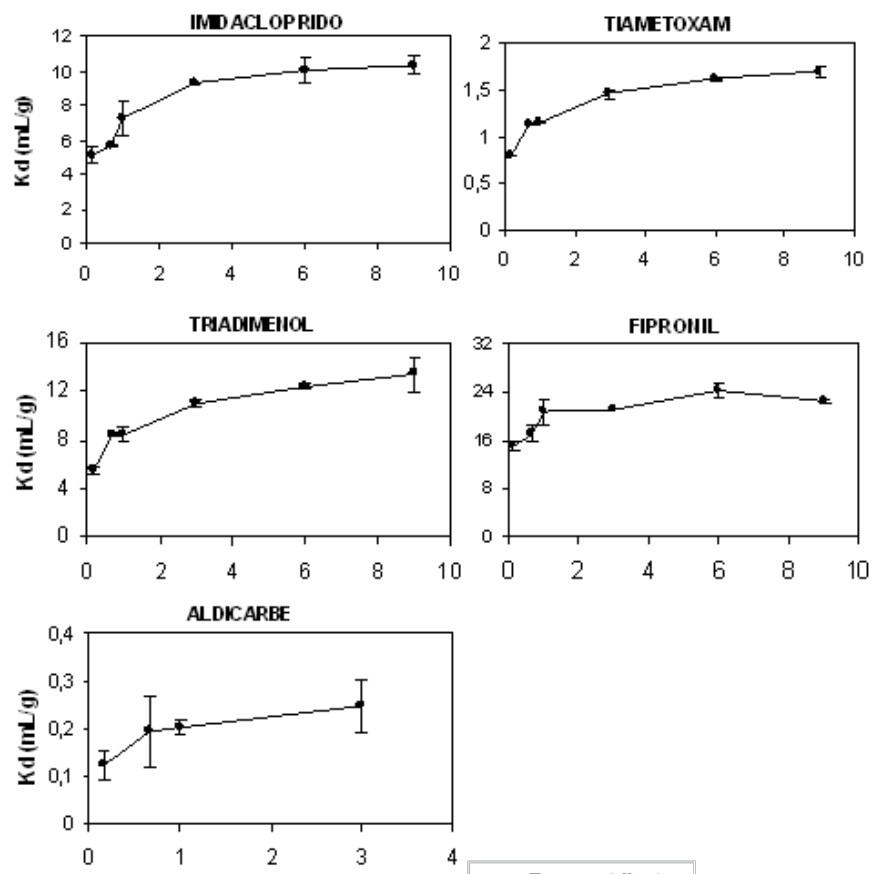

Tempo (dias) 
Constatou-se sorção inicial relativamente rápida para todos os pesticidas, com tendência ao equilíbrio após 8 horas de agitação. Em outros trabalhos envolvendo diversos pesticidas não-iônicos e diferentes solos (FELSOT e DAHM, 1979; CAPRI et al., 2001) também foi verificada rápida sorção inicial dos compostos, com o equilíbrio sendo atingido com menos de 4 horas de agitação. Observouse aumento apreciável no valor de Kd do tiametoxam, do triadimenol e do aldicarbe após 24 horas de agitação em relação ao valor obtido após 8 horas. As razões desse aumento não são claras e podem ter sido reflexo de variação experimental. URZEDO et al. (2006) constataram equilíbrio de sorção desse pesticida em solo da classe Latossolo Vermelho - Amarelo Distroférrico em 4 horas de agitação. Da mesma forma, CARVALHO, RIGITANO e LIMA (2002) verificaram que o equilíbrio de sorção do triadimenol em amostras de solo da classe Podzólico Vermelho - Amarelo também foi atingido em 4 horas.

A variação na proporção de solo:solução aquosa ( $\mathrm{g} \mathrm{mL}-1$ ) na faixa de 0,5:10 a 5:10 não resultou em expressiva variação nos valores de $\mathrm{Kd}$ dos compostos (Tabela 2). Os valores de $\mathrm{Kd}$ aumentaram para a maioria dos compostos com a elevação na proporção solo:solução (aumento inferior a 20\%). CELIS et al. (1999) observaram aumento no valor de Kd do fungicida triadimefon inferior a $25 \%$ quando a proporção de solo:solução variou de 1:10 a 4:10 (g mL-1).

\section{TABELA 2 - VALORES DE Kd OBTIDOS COM A VARIAÇÃO DA MASSA DE SOLO E O VOLUME DA SOLUÇÃO APLICADA FIXADO EM $10 \mathrm{~mL}$}

\begin{tabular}{lcccc} 
Pesticida & \multicolumn{5}{c}{ Massa de solo (g) } \\
\cline { 2 - 5 } & $\mathbf{0 , 5}$ & $\mathbf{1}$ & $\mathbf{2}$ & $\mathbf{5}$ \\
\hline imidacloprido & 3,53 & 3,43 & 3,52 & 3,75 \\
tiametoxam & 0,97 & 1,3 & 1,15 & 1,18 \\
triadimenol & 6,64 & 6,59 & 7,39 & 7,78 \\
fipronil & 22,3 & 22,94 & 23,58 & 23,45 \\
Aldicarbe & 0,36 & 0,37 & 0,36 & 0,32 \\
& & & & \\
\hline
\end{tabular}

\subsection{COMPARAÇÃO DOS COEFICIENTES DE SORÇÃO OBTIDOS PELOS DIFERENTES MÉTODOS}

Os valores de $\mathrm{Kd}$ dos compostos obtidos pelo método de incubação foram maiores do que aqueles constatados pelo método de agitação, com exceção do fipronil (Tabela 3). Esses resultados indicam que a agitação por 24 horas não foi suficiente para que o equilíbrio de sorção nos sítios menos acessíveis fosse alcançado. No caso do fipronil, devido ao seu alto coeficiente de sorção, quantidades relativamente baixas do mesmo permaneceram na fase aquosa para subseqüente sorção nos sítios menos acessíveis. CAPRI et al. (2001) constataram valores de Kd do imidacloprido e pirimetanil, determinados pelo método da incubação superiores aos valores obtidos pelo método da agitação. Esses autores atribuíram o aumento no valor de Kd dos compostos em função do tempo de incubação a dois fatores: presença de sítios de sorção internos e menos acessíveis na massa de solo, em que a situação de equilíbrio é atingida mais lentamente; e degradação dos compostos no solo, tendo assumido que a degradação dos compostos na fase aquosa teria sido mais rápida do que sua dessorção. No presente trabalho, à exceção do aldicarbe, 
os compostos revelaram-se muito estáveis de maneira que o aumento observado nos valores de Kd não pode ser atribuído à degradação.

TABELA 3 - VALORES DE Kd DOS PESTICIDAS, OBTIDOS PELOS MÉTODOS DE AGITAÇÃO (24h) E INCUBAÇÃO (9d) EM AMOSTRAS DE ARGISSOLO VERMELHO - AMARELO DISTRÓFICO TÍPICO

\begin{tabular}{lcc} 
Pesticidas & Kd de agitação & Kd de incubação \\
\hline tiametoxam & 1,16 & 1,7 \\
imidacloprido & 4,32 & 10,36 \\
triadimenol & 8,3 & 13,6 \\
Fipronil & 23,58 & 22,38 \\
\hline
\end{tabular}

Uma das conseqüências das diferenças nos valores de $\mathrm{Kd}$ dos compostos determinados pelos métodos de incubação e de agitação é que a previsão da lixiviação de pesticidas em solos sofre forte influência do valor de $\mathrm{Kf}$ ou $\mathrm{Kd}$ do composto. Os modelos matemáticos e programas computacionais desenvolvidos para a simulação da lixiviação de pesticidas em solos requerem, em geral, informações sobre o valor de $\mathrm{Kd}$ ou $\mathrm{Kf}$ dos compostos, normalmente determinados pelo método de agitação por 24 horas. Os resultados obtidos no presente trabalho mostraram que os valores de $\mathrm{Kd}$ dos compostos, determinados pelo método de incubação, aumentaram apreciavelmente até o terceiro dia de incubação. Como tal método é mais próximo das condições de campo, tais resultados indicam que a utilização de parâmetros de sorção dos compostos obtidos pelo método de incubação pelos simuladores da lixiviação de pesticidas em solos tornaria esses modelos mais eficientes na análise de risco de contaminação de águas subterrâneas com resíduos de pesticidas.

\section{CONCLUSÃO}

A sorção dos pesticidas imidacloprido, tiametoxam e triadimenol em material do horizonte $A$ de solo da classe Argissolo Vermelho-Amarelo Distrófico típico revelou-se um processo lento, com tendência ao equilíbrio de sorção sendo verificado somente após 3 dias de incubação de amostras desse solo com os compostos. No caso do inseticida fipronil, composto menos polar do que os demais estudados, a tendência ao equilíbrio de sorção foi observada a partir de um dia de incubação.

Os valores dos coeficientes de sorção $(\mathrm{Kd})$ dos compostos, obtidos pelo método de incubação foram maiores do que aqueles constatados pelo método de agitação, com exceção do fipronil que apresentou valores semelhantes pelos dois métodos.

\section{ABSTRACT}

INFLUENCE OF TIME ON THE SORPTION OF NON-IONIC PESTICIDES IN AN ARGISOL

The influence of time on the sorption of non-ionic pesticides was investigated on soil material from the A horizon of an Argisol - typical Distrophic Yellow. Five pesticides, with extensive polarities, expressed by their n-octanolwater partition coefficients (Kow), were studied. The distribution coefficients (Kd) of the compounds in the soil 
were determined using the incubation and agitation methods. The quantitative determinations of the pesticides were performed by high performance liquid chromatography. The results showed that sorption of the compounds in the studied soil, as measured by the incubation method, is a relatively slow process. Tendency to equilibrium being observed only after three days was verified with the exception of the least polar compound studied, whose sorption equilibrium was observed after one day of incubation. Using the agitation method, the tendency of sorption equilibrium was demonstrated following the first hours for all pesticides, with their Kd values, as determined by this method, being lower than those obtained by the incubation method.

KEY-WORDS: ALDICARB; PHIPRONIL; IMIDACLORPRID; THIAMETHOXAM; TRIADIMENOL; SOIL; SORPTION; LEACHING.

\section{REFERÊNCIAS}

1 BRIGGS, G.G. Theoretical and experimental relationship between soil adsorption, octanol-water partition coefficients, water solubilities, bioconcentration factors, and the parachor. Journal of Agriculture and Food Chemistry, Washington, v. 29, n. 6, p. 1050-1059, 1981.

2 BROMILOW, R.H.; BRIGGS, G.G; WILLIANS, J.H.; SMELT, J.H.; TUINSTRA, L.G.M.T.; TRAAG, W.A. The role of ferrous ions in the rapid degradation of oxamil, methomyl and aldicarb in anirobic soils. Pesticide Science, England, v. 17, p. 535-547, 1986.

3 CAPRI, E.; CAMISA, M.G.; FLORES-CÉSPEDES, F.; GLASS, C.R.; GONZALEZ-PRADAS, E.; TREVISAN, M. Imidacloprid and pyrimethanil soil sorption. Agronomie, Ulis Cedexa, v. 21, n. 1, p. 57-64, 2001.

4 CARVALHO, R.F. de; RIGITANO, R.L. de O.; LIMA, J.M. de. Sorção e degradação do fungicida triadimenol em solos representativos do Município de Lavras - MG. Ciência e Agrotecnologia, Lavras, v. 26, p. 332-341, 2002.

5 CELIS, R.; KOSKINEN, W.C.; HERMOSIN, M.C.; CORNEJO.J. Sorption and desorption of triadimefon by soils and model soil colloids. Journal of Agriculture and Food Chemistry, Washington, v. 47, n. 2, p. 776-781, Feb.1999.

6 COX, L.; KOSKINEN, W.C.; YEN, P.Y. Changes in sorption of imidacloprid with incubation time. Soil Science Society of America Journal, v. 62, p. 342-347, Mar./Apr.1998.

7 DOMAGALSKI, J. L.; DUBROVXKY, N. M. Pesticide residues in ground water of the San Joaquim Valley, California. Journal of Hidrology, Amsterdam, v. 130, n.1/4, p. 299-338, Jan.1992.

8 FELSOT, A.; DAHM, P.A. Sorption of organophosphorus and carbamate insecticides by soil. Journal of Agricultural and Food Chemistry, Washington, v. 27, n. 3, p. 557-563, 1979.

9 KOSKINEN, W.C.; HARPER, S.S. The retention process: mechanisms. In: CHENG, H. H. et al. (Ed.). Pesticides in the soil environment: processes, impacts and modeling. Madison: Soil Science Society of America, 1990. p. 51-78.

10 PIASAROLO, L.; RIGITANO, R. L.O.; GUERREIRO, M. C. Influência da polaridade de pesticidas não-iônicos sobre sua sorção em um Latossolo. Ciência e Agrotecnologia, Lavras, v. 32, n. 6, p. 100-108, 2008.

11 PIFFER, R.; RIGITANO, R.L.O. Lixiviação e degradação do inseticida aldicarbe em dois diferentes solos. Ciência e Prática, Lavras, v. 15, n. 4, p. 355-363, out./dez. 1991.

12 RIGITANO, R.L.O.; GOUVÊA, A. V. Contaminação de manancial hídrico com resíduos de inseticidas em Maria da Fé - MG. In: CONGRESSO BRASILEIRO DE ENTOMOLOGIA, 15., 1995, Caxambú. Resumos... Lavras: SEB/UFLA, 1995. 485 p.

13 SCHWARZENBACH, R.P.; GSCHWEND, P.M.; IMBODEN, D.M. Environmental organic chemistry. New York: J. Wiley \& Sons, 1993. 681 p.

14 TOMLIN, C.D.S. (Ed.). The pesticide manual. 12 ${ }^{\text {th }}$ ed. Bath, UK: British Crop Protection Council, 2000. $1250 \mathrm{p}$.

15 URZEDO, A. P. F. M.; RIGITANO, R.L.O.; LIMA, J. M.; CASTRO, N. R. A. Sorção do inseticida tiametoxam em amostras de solos da região de Lavras - MG. Pesticidas: revista de ecotoxicologia e meio ambiente, Curitiba, v.16, p. 71-80, jan./dez. 2006. 
16 VAN RAIJ, B.; QUAGGIO, J.A.; CANTARELLA, H.; FERREIRA, M. E.; LOPES, A. S.; BATAGLIA, O. C. Análise química do solo para fins de fertilidade. Campinas: Fundação Cargill, 1987. 170 p.

17 WALKER, A. Evaluation of a simulation model for prediction of herbicide movement and persistence in soil. Weed Research, v. 27, p. 143-152, 1987. 